\title{
Numerical Investigation of Turbulent Flow inside a Cubical Cavity with Heat Transfer from the Top
}

\author{
Vitaly Haslavsky ${ }^{1}$, Helena Vitoshkin ${ }^{2}$ \\ ${ }^{1}$ Azrieli College of Engineering \\ 26 Yaakov Shreibom Street, Jerusalem, Israel \\ vitaliha@jce.ac.il, elenav@volcani.agri.gov.il \\ ${ }^{2}$ Agricultural Research Organization, The Volcani Center \\ P.O.Box 6, 5025001, 68 Dereh Hamacabim, Rishon lesion, Israel
}

\begin{abstract}
Numerical investigation of natural convection in cavities with heating from below, adiabatic sidewalls, and variable heat flux in combination with radiative and convective heat transfer on the top is the framework of this research. The goal is to develop a computational model for investigation of overall heat transfer coefficients through different types of thermal screens and their integration with greenhouse covers in order to improve greenhouse insulation. The model was developed by applying hot box methodology, and calculations are validated by published results for convective flow in the cubical cavity model. A greenhouse covering combined with moveable thermal screens can block a high amount of IR radiation. Moreover, thermal screens in combination with cooling/heating and dehumidification systems may provide desirable conditions in a closed greenhouse and reduce year-round energy consumption. By significantly reducing fossil energy consumption, this design contributes to sustainability as well. The numerical modeling supported by laboratory experiments is not only enhances understanding of the greenhouse insulation material processes but also of other material processing and technological applications, including high-temperature technologies, solar collectors, and residential and industrial construction.
\end{abstract}

Keywords: Natural convection, Computational Fluid Dynamics (CFD), turbulent flow, heat transfer, greenhouse Microclimate.

\section{Introduction}

One of the major objectives of intensive greenhouse production is to provide environmental conditions conducive to maintaining the growth cycle throughout the year and in different regions around the world. Agriculture, in greenhouses as in open fields, faces problems in resisting strong winds, rain, hail, snow, and other devastation risks. Despite all these obstacles, the crop industry must supply high-quality products on a daily basis, according to market commitments. In order to receive predicted yields throughout the year, seedlings are planted continuously in different environmental conditions (sprouts are usually grown in isolation and transplanted in a greenhouse).

The temperature of the air and soil in the greenhouse is very important for the cultivation of any crop and therefore, it is necessary to know not only the temperature limits for specific plants but also how to adjust the indoor conditions to the local climate outside at different seasons and periods of the day. Day treatment is entirely different from night care. During the day, photosynthesis is emitted along with an excess of heat. At night, it is cold (the day/night temperature difference is about $10^{\circ}-15^{\circ} \mathrm{C}$ ) with high humidity (in desert areas, humidity can reach up to $70 \%$ in summer and $90 \%$ in winter). In warm countries, such as the Mediterranean region, including Israel, cultivation in a greenhouse usually occurs in winter. Planting takes place in September with a growing season through the end of March, while screen-houses are used in summer. This growth process is not optimal, and methods for improving production are needed.

Analysis of the conventional environmental control treatments within the greenhouse can help to determine how to advance the production process. Traditional systems include natural or forced ventilation during the day and heating at night with fan heater pipes or heated sleeves, evaporative cooling systems (known as fan and pad cooling), and active dehumidification systems [1-8]. However, contemporary systems are very energy-intensive, reducing cost-effectiveness and increasing the amount of environmental pollution. 


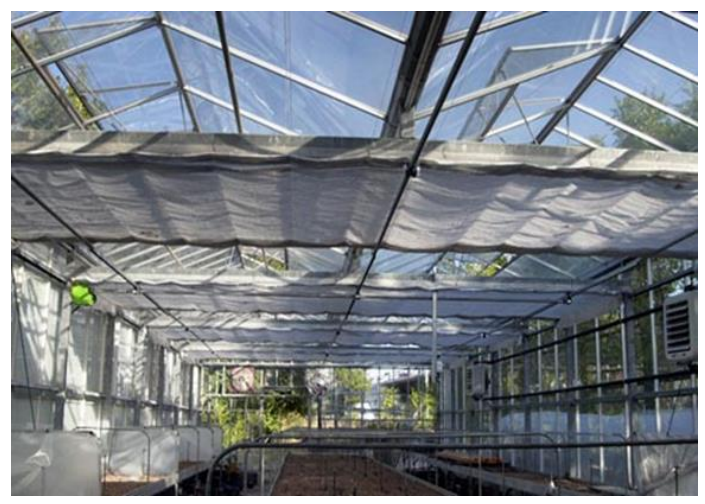

Fig. 1: Shading with thermal screens inside a typical greenhouse.

Energy-savings screens, or thermal screens, are commonly used in closed greenhouses (Figure 1). These screens roll in and out easily in order to provide stable climatic conditions, such as internal shading and insulation (Error! Reference source not found.). In general, the energy-saving properties of thermal screens are related to buoyancy, diffusion and convection heat transfer, air permeability, and humidity transfer [9-11]. However, mechanical dehumidification systems help to prevent condensation due to excess humidity and therefore eliminate the need for air permeability and extensive ventilation [12-14]. In this case, knitted permeable screens are no longer required, since whole sheets of material, which are much cheaper and easier to manufacture, may be used. Thus, material emissivity becomes the main factor when choosing a thermal screen for reducing energy loss. The energy loss depends on the exchange of heat radiation among crops, dehumidification systems, greenhouse screens, coverings, and the sky [15]. Depending on the time of day and weather conditions, unrolled screens with a sun/sky reflective outer layer provide efficient insulation; in particular, they retain coolness in the summer (during the day) and warmth in winter (overnight). At night, using an IR reflecting inner layer, part of the thermal radiation from inside the warm greenhouse can be absorbed and emitted by the screen material. Thus, combining (by collapsing or expanding) the different types of screens depending on the external weather conditions, affords proper control of light, temperature, and humidity, maintaining the optimal levels for growing with significant savings in energy. Moreover, using several layers of the double-sided aluminized film provides nearly $99 \%$ insulation in the summer, reducing solar gain and guaranteeing excellent insulation [16-20].

The main objective of the paper is the computational modeling of the overall heat transfer coefficient for multilayer thermal screens to more complicated models that include most, if not all, physical phenomena involved in the process (Figure 2). To achieve this purpose, a computational model is developed, including the simulation of a turbulent internal flow in combination with radiative and convective heat transfer through several layers of different screen materials. Turbulent flow characteristics and temperature fields are extensively measured by techniques that have already been successfully applied in previous experiments. To ensure a better comparison with the computational modeling, several unknown or poorly known parameters is measured by conducting separate experiments. 


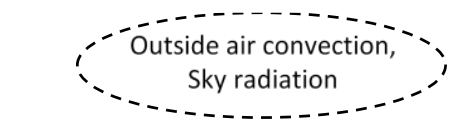

Heat flux through the multilayer screens

(radiation and convection, Equation 4)

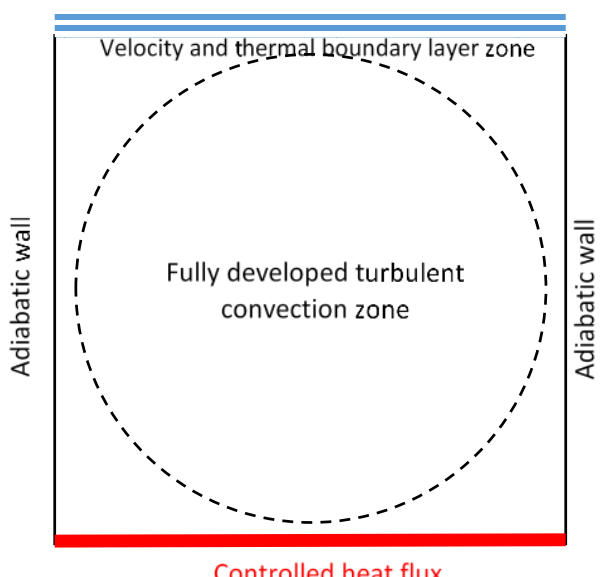

Fig. 2: Sketch of the problem for mathematical formulation. Heat transfer by conduction and radiation must be taken into account everywhere. In the case of semi-transparent and/or opaque screens, a model of radiation in the participating media must be included.

\section{Results}

\section{1. Convective flow in a cubical cavity}

At the first stage, we consider a fluid-filled cavity of width $\mathrm{W}$, depth $\mathrm{D}$, and height $\mathrm{H}$, with two opposing vertical walls, maintained at fixed temperatures $T_{h}$ and $T_{c}$, with the other two walls being insulated. Due to buoyancy, fluid motion is induced in the cavity, and depending on the cavity geometry, the working fluid, and the temperature difference, $\Delta \mathrm{T}=\mathrm{T}_{\mathrm{h}}-\mathrm{T}_{\mathrm{c}}$. In terms of dimensional analysis, the representative parameters are the geometrical aspect ratios $\left(A_{x}=W / H\right.$ and $\left.A_{y}=D / H\right)$, the Prandtl number $\operatorname{Pr}=v / \kappa$ (here $v$ and $\kappa$ are, respectively, the kinematic viscosity and thermal diffusivity), and the Rayleigh number $\mathrm{Ra}=\left(\mathrm{g} \beta \Delta \mathrm{TH}^{3}\right) /(v \kappa)$ (g is the gravitational constant and $\beta$ is thermal expansion coefficient).

The cavity is filled with air $\left(\operatorname{Pr}=0.71, \mathrm{Ra}=1.5 \times 10^{9}\right)$, and its size is fixed to $\mathrm{H}=1 \mathrm{~m}$, with $\mathrm{A}_{\mathrm{x}}=1$ and $\mathrm{A}_{\mathrm{y}}=0.32$. Therefore, airflow in the cavity will depend only on the temperature difference, $\Delta \mathrm{T}$. For the flow regime at a high Rayleigh number, large-scale computations such as DNS and LES are now becoming increasingly feasible. An RSM model with SST lowReynolds correction at the walls was used in our numerical simulation. The SIMPLE algorithm is used for coupling between the velocity and pressure fields. All the terms involved in the balance equations are evaluated with second-order accurate centered schemes, and a QUICK scheme is used for the nonlinear terms of the momentum equations. The solver runs in parallel mode utilizing the domain decomposition MPI directives with the number of CPUs ranging from 16 to 32 .

Values of the averaged Nusselt number along with the hot wall are reported in Table 1 for the different cases considered. Two methodological approaches have been adopted to investigate the airflow at high Rayleigh numbers in this cavity: experimental measurements on the one hand and 3D numerical simulations on the other. It is shown that LES and DNS models overestimate the heat transfer [21]. Our 2D RMS model predicts results closer to the experiment (Figure 3); simulation data show the same profile tendencies as in the experiments. We assume that at high Rayleigh number, the heat transfer near the bottom and top walls occurs at a thin boundary layer near the wall (Figure 2), and a large number of grids are required for an accurate prediction of such a flow. 
Table 1: Comparison of the averaged Nusselt number along the hot wall median line, $\mathrm{Nu}$, for temperature imposed conditions on the horizontal walls. Superscribes: 1 - 2D grid $1000^{2} ; 2$ - 2D grid $300^{2}$.

\begin{tabular}{|c|c|c|c|}
\hline 3D LES & 3D DNS & RSM (our case) & Experiment \\
\hline 57.5 & 58 & $55.4^{1}, 55.8^{2}$ & 54 \\
\hline
\end{tabular}
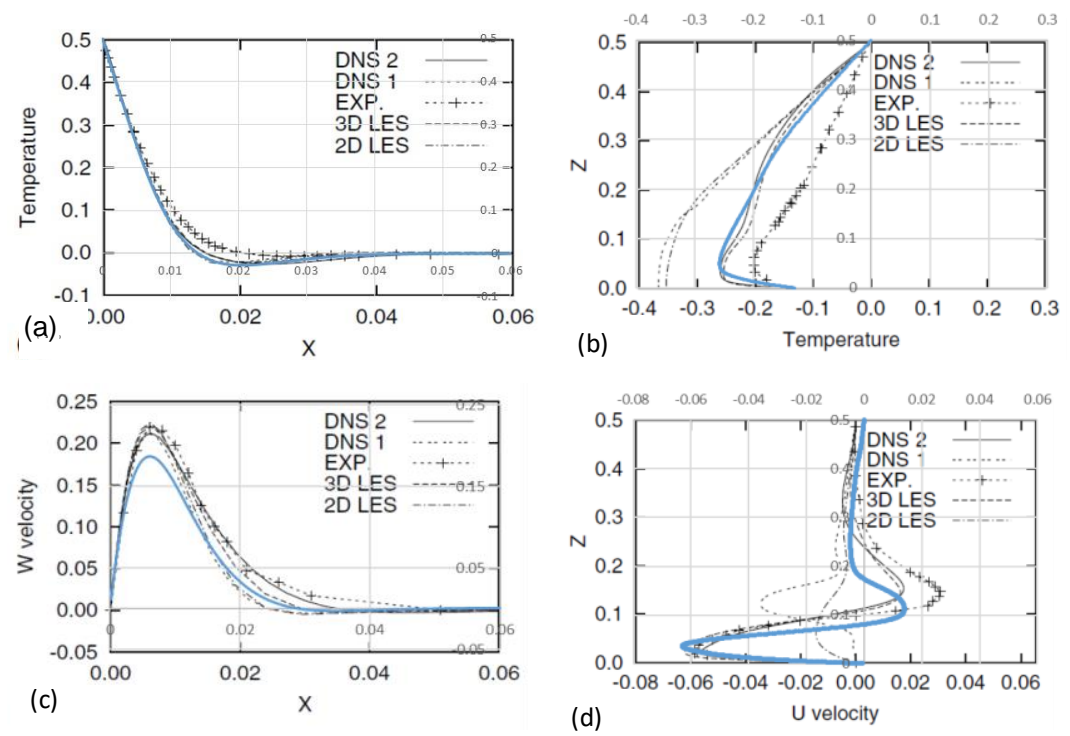

Fig. 3: Validation of our numerical model (blue lines) with results published by Salat et al., 2004. These results correspond to numerical and experimental investigation of turbulent natural convection inside a cubic cavity with temperature differences between vertical walls. Example of temperature and velocity profiles at mid-height $(\mathrm{a}, \mathrm{c})$ and mid-width $(\mathrm{b}, \mathrm{d})$ planes of the cavity for

$$
R a=1.5 \times 10^{9} \text {. }
$$

\section{2. Natural convection within a hotbox of a $1: 1$ aspect ratio}

We consider natural convection within a hotbox of a 1:1 aspect ratio where the bottom wall is heated, and the upper wall is cooled (Figure 4). A non-slip condition is imposed at the lateral boundaries. A suitable mesh has been adopted to make a balance between computational accuracy and efficiency. The 128 non-uniform grids are generated in the vertical direction, and the smallest grid size near the walls is $\Delta y / H=1 \times 10^{-4}$, where $\mathrm{H}$ is the vertical distance between two horizontal walls. In the lateral direction 256 uniform grids are generated. Calculations are performed for Rayleigh number is $4 \times 10^{9}$, the temperature of the bottom wall is $333 \mathrm{~K}$, and the temperature of the top wall is $294 \mathrm{~K}$. The grid independence tests show that the given mesh resolutions are adequate. Figure $4 \mathrm{c}$ shows the predicted mean average Nusselt number at the heated bottom wall. 
(a)

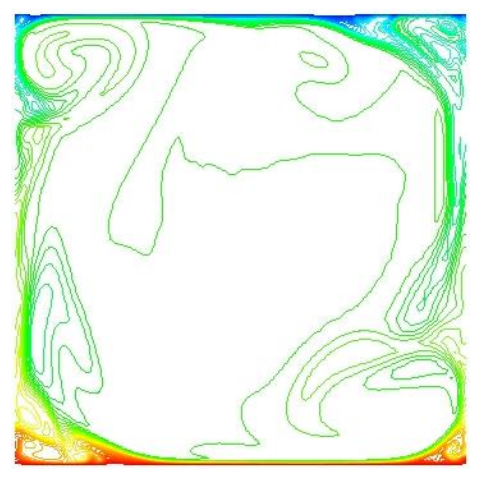

(b)

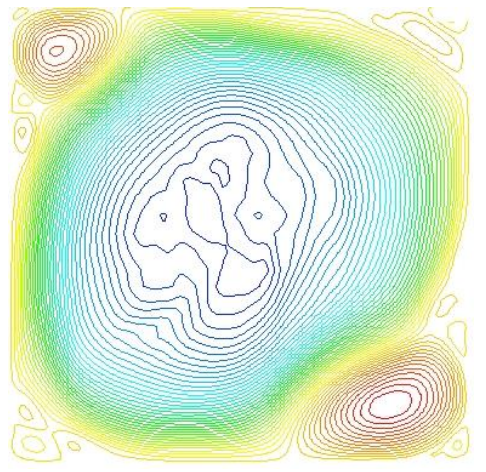

(c)

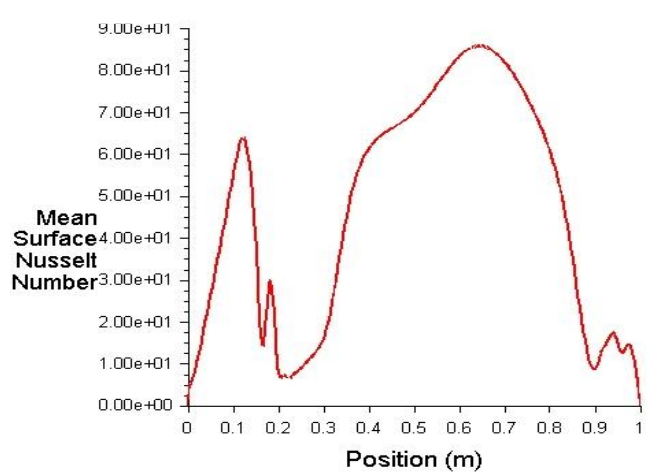

Fig. 4. Preliminary numerical model a) isothermals, b) streamlines and c) horizontal surface Nusselt number of natural convection inside a cavity with heating from below for cubic cavity for $R a=4 \times 10^{9}$.

\section{3. One-dimensional steady-state energy balance}

Given boundary conditions (wind, solar radiation, temperature) that remain constant over the response time of the thermal screen, the temperature $\mathrm{T}$ of each layer is determined by the conditions that no net energy is absorbed or released by any layer. An individual sheet is denoted by subscription $i$. Sheets are numbered from the inside out. The radiative flux leaving the individual sheet surface is denoted by $q_{i ; i+1}^{r}$, or $q_{i ; i-1}^{r}$, and the convective flux at the individual layer surfaces is denoted by $q_{i ; i+1}^{c}$, or $q_{i ; i-1}^{c}$, here $i+1$, and $i-1$ are indexes of neighboring layers. For a system of $\mathrm{n}$ layers, the temperature of each sheet is desired. Individually, a system of $n$ nonlinear equations for the $\mathrm{n}$ sheets temperature is solved. The behavior of a surface with radiation incident upon it can be described by the following quantities: $\alpha$ - absorptance - a fraction of incident radiation absorbed; $\rho$ - reflectance - a fraction of incident radiation reflected; and $\tau$ - transmittance - a fraction of incident radiation transmitted. In the case of thermo-screens used in greenhouses, $\alpha+\rho+\tau=1$. We obtain the expression for heat transfer between two planar thermo-screens:

$$
q_{i, i+1}^{r}=\frac{\sigma\left(T_{i}^{4} \beta_{i+1}-T_{i+1}^{4} \beta_{i}\right)}{\beta_{i+1} / \epsilon_{i}+\beta_{i} / \epsilon_{i+1}-\beta_{i} \beta_{i+1}}
$$

where $\sigma$ is the Stefan-Boltzmann constant and $\beta_{i}$ is following ratio:

$$
\sigma=5.67 \cdot 10^{-8} \mathrm{~W} / \mathrm{m}^{2} K^{4}, \beta_{i}=\tau_{i} / \epsilon_{i}, \beta_{i+1}=\tau_{i+1} / \epsilon_{i+1}, \epsilon_{i}=\alpha_{i}
$$

Decompose Equation 1 to the form:

$$
q_{i, i \pm 1}^{r}=\gamma_{i}\left(T_{i} \beta_{i \pm 1}^{1 / 4}-T_{i \pm 1} \beta_{i}^{1 / 4}\right)
$$

where $\gamma_{i}$ is a function of temperatures of $i ; i-1$; and $i+1$ screens and their properties. An energy balance is performed at each screen layer, and equations are written as: 


$$
\begin{gathered}
q_{i, i-1}^{r}-q_{i, i+1}^{r}+q_{i, i-1}^{c}-q_{i, i+1}^{c}+\sum_{\substack{j<i-1 \\
j>1+1}} q_{j}^{t r}=0 \\
q_{i, i-1}^{c}=h_{i, i-1}\left(T_{i}-T_{i, i-1}^{a i r}\right), \quad q_{i, i+1}^{c}=h_{i, i+1}\left(T_{i}-T_{i, i+1}^{a i r}\right) \\
q_{j}^{t r}=q_{i, j}^{r} \prod_{\substack{j<i-1 \\
j>1+1}} \tau_{j}
\end{gathered}
$$

where $q^{t r}$ is radiative heat transmittance, calculated in accordance with Equation 1 , and $h_{i ; i \pm l}$ is a convective heat transfer coefficient at sheet side facing to sheet $i \pm l$.

The final form of the linearized Equation 4 specific to sheet $i$ may be written as:

$$
\alpha_{i} T_{i}=c_{i} T_{i-1}+d_{i} T_{i+1}+b_{i}, i=1 \ldots n
$$

in matrix form:

$$
A \cdot \boldsymbol{T}=B, \quad A=\left(\begin{array}{ccccc}
a_{1,1} & a_{1,2} & & \\
& a_{i, i-1} & a_{i, i} & a_{i, i+1} & \\
& & & a_{n, n-1} & a_{n, n}
\end{array}\right), B=\left(b_{i}\right)
$$

where $a_{i, i}=a_{i} ; a_{i, i-1}=c_{i} ; a_{i, i+1}=d_{i}$

and

$$
\begin{gathered}
c_{i}=\gamma_{i-1} \beta_{i+1}^{1 / 4}+h_{i, i-1} \\
d_{i}=\gamma_{i+1} \beta_{i}^{1 / 4}+h_{i, i+1} \\
a_{i}=c_{i}+d_{i} \\
b_{i}=h_{i, i-1} T_{i, i-1}^{a i r}+h_{i, i+1} T_{i, i+1}^{a i r}+\sum_{\substack{j<i-1 \\
j>1+1}} q_{j}^{t r}
\end{gathered}
$$

The linear system (6) is solved by a Tridiagonal matrix algorithm (TDMA). Matrix A is diagonally dominant. Therefore, the TDMA procedure converges for any initial guess $T_{0}$.

Thus, we developed a calculation procedure for heat transfer through screens in which three diagonal matrices are used. Only neighboring layers $i-1$ and $i+1$ explicitly form down and upper diagonals of the linear system matrix. Interaction $i$ layer with transparent layers is calculated implicitly. The linear system matrix is solved by TDMA. Our procedure thus demonstrates higher convergence; only two- or three iterations are needed to resolve the nonlinearity of the radiative heat flux and to be used as UDF for FLUENT implementation.

\section{Conclusion}

The main objective of the research is the development of a computational model as an extension of the laboratory hotbox model of the overall heat transfer coefficient for multilayer thermal screens. The computational model includes simulation of a turbulent internal flow in combination with radiative and convective heat transfer through several layers of different screen materials. Turbulent flow characteristics and temperature fields are investigated, and results are validated via published results for convective flow in the cubical cavity. Horizontal surface Nusselt number of natural convection 
inside a cavity with heating from below for cubic cavity was calculated. The robust calculation procedure for onedimensional steady-state energy balance is presented.

Successful computational modeling can be helpful for further development of greenhouse climate control, especially in terms of optimizing greenhouse energy, minimizing cost, and customizing for specific plant cultivation. The results of the

study will help growers to understand more about energy-saving screen properties and allow them to make more informed investment decisions.

\section{References}

[1] C. Zabeltitz "Energy efficient greenhouse designs for Mediterranean countries," Plasticulture, vol. 96, no. 4, pp. 6-16, 1992.

[2] V. Elsner, D. Briassoulis, D. Waaijenberg, A. Mistriotis, C. V. Zabeltitz, J. Gratraud, G. Russo, and R. Suay-Cortes, "Review of structural and functional characteristics of greenhouses in European Union countries: Part I, design requirements," Journal of Agricultural Engineering Research, vol. 75, no. 1, pp. 1-16, 2000.

[3] A. Baille, J. C. López, S. Bonachela, M. M. González-Real, and J. I. Montero, "Night energy balance in a heated lowcost plastic greenhouse," Agricultural and forest meteorology, vol. 137, no. 1-2, pp. 107-118, 2006.

[4] N. Castilla, "Greenhouse technology and management," Cabi, 2013.

[5] J. Parra, E. Pérez, J. Baeza, I. Montero, and B. J. Bailey, "Natural ventilation of parral greenhouses," Biosystems engineering, vol. 87, no. 3, pp. 355-366, 2004.

[6] L. Okushima, S. Sase, and M. Nara. "A support system for natural ventilation design of greenhouses based on computational aerodynamics," International Symposium on Models for Plant Growth, Environmental Control and Farm Management in Protected Cultivation, vol. 248, pp. 129-136. 1988.

[7] J. Tanny, B. Yakubov, "Experimental study of a double-diffusive two-layer system in a laterally heated enclosure," International Journal of Heat and Mass Transfer, vol. 42, no. 19, pp. 3619-3629, 1999.

[8] C. Kittas, T. Boulard, and G. Papadakis, "Natural ventilation of a greenhouse with ridge and side openings: sensitivity to temperature and wind effects," Transactions of the ASAE, vol. 40, no. 2, pp. 415-425, 1997.

[9] S. Hemming, J. Balendonck, J. A. Dieleman, A. De Gelder, F. L. K. Kempkes, G. L. A. M. Swinkels, P. H. B. De Visser, and H. F. De Zwart, "Innovations in greenhouse systems-Energy conservation by system design, sensors and decision support systems," Acta horticulturae, vol. 1170, pp. 1-15, 2017.

[10] J. Nijskens, J. Deltour, S. Coutisse, and A. Nisen, "Radiation transfer through covering materials, solar and thermal screens of greenhouses," Agricultural and forest Meteorology, vol. 35, no. 1-4, pp. 229-242, 1985.

[11] K. Abak, A. Bascetincelik, N. Baytorun, Ö. Altuntas, and H. H. Öztürk, "Influence of double plastic cover and thermal screens on greenhouse temperature, yield and quality of tomato," II Symposium on Protected Cultivation of Solanacea in Mild Winter Climates, vol. 366, pp. 149-154, 1993.

[12] A. Arbel, M. Barak, and A. Shklyar, "Method and system for heating and dehumidifying," International Application no. PCT/IL2008/001449, 2008.

[13] A. Arbel, M. Barak, A. Shklyar, G. Lidor, Y. Elad, M. Sofer, H. Yehezkely, D. Shmueli, A.Slepoy and L. Ganot, "Performance of combined heating and dehumidification system for greenhouses," Acta Hort., vol. 952, pp. 449-456, 2012.

[14] J. B. Campen, F. L. K. Kempkes, and G. P. A. Bot, "Mechanically controlled moisture removal from greenhouses," Biosystems engineering, vol. 102, no. 4, 424-432, 2009.

[15] J. Nijskens, J. Deltour, S. Coutisse, and A. Nisen, "Radiation transfer through covering materials, solar and thermal screens of greenhouses," Agricultural and forest Meteorology, vol. 35, no. 1-4, pp. 229-24, 1985.

[16] B. J. Bailey, "Thermal screens for reducing heat losses from glasshouses," Acta Hort. (ISHS), vol. 70, pp. $26-34,1977$.

[17] B. J. Bailey, "Control strategies to enhance the performance of greenhouse thermal screens," J. Agric. Engin. Res., vol. 40, pp. 187-198, 1988.

[18] F. Geoola, Y.Kashti, A. Levi and R. Brickman, "A study of the overall heat transfer coefficient of greenhouse cladding materials with thermal screens using the hot box method," Polymer Testing, vol. 28, pp. 470-474, 2009. 
[19] J. Meyer, "Energy saving with mobile thermal screens," Acta Hort. (ISHS), vol. 115, pp. 677-684, 1981.

[20] H. Vitoshkin, M. Barak, C. Shenderey, V. Haslavsky, A. Arbel, "Improving greenhouse insulation through multilayer thermal screens using the hot box method," 5th World Congress on Mechanical, Chemical, and Material Engineering (MCM'19) Proceedings, Lisbon, Portugal, 2019.

[21] F. Sebilleau, R. Issa, S. Lardeau, \& S. P. Walker, "Direct Numerical Simulation of an air-filled differentially heated square cavity with Rayleigh numbers up to 1011," International Journal of Heat and Mass Transfer, vol. 123, pp. 297 319. 2018. 